
\title{
25 Research Square \\ Doula Support in Office Hysteroscopy: Results From A Pilot Study
}

Rocio Montejo ( $\nabla$ rocio.montejo.rodriguez@vgregion.se )

Sjukhus i Väst

Jonas Hermansson

Angered Närsjukhus

Lena Sandin Wranker

University of Gothenburg: Goteborgs Universitet

Louise Danielsson

Angered Närsjukhus. University of Gothenburg: Goteborgs Universitet

\section{Research}

Keywords: Anxiety, Doula Support, Human Support, Office Hysteroscopy, Pilot Study

Posted Date: November 18th, 2020

DOl: https://doi.org/10.21203/rs.3.rs-106947/v1

License: (c) (1) This work is licensed under a Creative Commons Attribution 4.0 International License. Read Full License

Version of Record: A version of this preprint was published at Clinical and Experimental Obstetrics \& Gynecology on January 1st, 2021. See the published version at https://doi.org/10.31083/j.ceog4804151. 


\section{Abstract}

Background This pilot study aimed to evaluate the feasibility of doula support in office hysteroscopy and the potential effectiveness of doula support during office hysteroscopy to reduce anxiety and pain.

Methods This study was designed as a two-armed pilot study, with an intervention and a control group, with participants allocated to each group in a 1:1 ratio. 30 of 58 patients were initially excluded from due to not could read Swedish or declining to participate in the study. Twenty-eight women with indications for office hysteroscopy were randomized to receive doula support (intervention) during the procedure or to routine care (control group). The data collected included general and gynecological data, Spielberg StateTrait Anxiety Inventory-S (STAI), and the Numeric Rate Scale (NRS) for pain intensity. Feasibility was measured in terms of successful office hysteroscopies, duration, and adverse events.

Results Success rates, duration, and adverse events were similar between the groups, with no differences in reported pain intensity. Both groups had high, comparable levels of anxiety before the procedure, whereas after the procedure, the doula group showed slightly increased anxiety while the control group showed slightly decreased anxiety. There was a significant difference between groups favoring the control group when comparing STAI-S mean score post-procedure but not when analysing the mean change across groups.

Conclusion Exploring a new take on doula support, this pilot study suggests that it may be feasible but not superior to routine care support in office hysteroscopy. Also, high anxiety levels may be more of an issue than pain during the procedure. Further investigation of the state and trait anxiety in different hysteroscopy populations and health care contexts is recommended

Trial Registration The study was registered in de Research Database Registry in Västra Götalandsregionen (Sweden) with number: 254161 on 10 November 2018.

\section{Background}

Office hysteroscopy is a widely used approach in gynaecology [1], however, effective pain management during the procedure is insufficient in about a third of patients [1], and pre-procedural anxiety is significantly higher than in those attending the gynaecologist's office for other reasons [2]. Nonpharmacological interventions to alleviate pain and anxiety are the variation of intrauterine pressure, transcutaneous electric nerve stimulation (TENS) [3-4] and listening to music [5] but the evidence is inconclusive [6]. Indeed, there is a lack of randomized studies exploring additional human support during office hysteroscopy.

The concept of a doula has expanded to encompass a person dedicated to providing physical, emotional, informational, and advocacy support to women during the pre-, peri-, and postnatal periods [7]. Doulas specialize in non-medical skills but do not perform clinical tasks or give medical advice. Recently, doula support has been evaluated in gynaecological procedures, such as surgical management of miscarriages 
and abortion, demonstrating high patient satisfaction [8-10]. Hence, it was hypothesized that the presence of a doula supporting the patient during office hysteroscopy would be beneficial. This pilot study aimed to evaluate a) the feasibility of doula support in office hysteroscopy and b) the potential effectiveness of doula support during office hysteroscopy to reduce state anxiety and pain.

\section{Methods}

\section{Study design}

This two-armed pilot study had an intervention and a control group, with participants allocated to each group in a 1:1 ratio. The CONSORT guidelines for pilot and feasibility study were adhered to [11]. The study was conducted in a gynaecological clinic in a community hospital located in southwest Sweden. Patients are referred to the hospital from primary care clinics, maternal health clinics or other hospitals in the region, and may also request care from the clinic by self-referral.

The study was registered in de Research Database Registry in Västra Götalandsregionen (Sweden) with number: 254161 on 10 November 2018.

\section{Participants}

Patients who attended the clinic between October 2018 to April 2020 were eligible for the study if they were at least 18 years old and had one of the following indications of office hysteroscopy: abnormal preor postmenopausal uterine bleeding; endometrial thickening or polyps; submucosal and some intramural fibroids; intrauterine adhesions; uterine malformations; retained intrauterine contraceptives or removal of foreign bodies. In cases of larger polyps $>2.5 \mathrm{~cm}$, myoma type $0>1.5 \mathrm{~cm}$ and myoma type 1 and 2 , participants were not eligible for office hysteroscopy or for this study.

The patients also needed to understand written Swedish to apprehend the study information and respond to the self-rated questionnaires. Exclusion criteria were viable intrauterine pregnancy, active pelvic infection (including genital herpes infection) or known cervical or uterine cancer, and poor ability to understand Swedish.

\section{Consent and randomisation}

Participants who accepted the invitation to participate signed a consent form and were randomly allocated to either the doula intervention or routine care. Based on week numbers (even/odd) when they were enrolled, the patients were alternated assigned to either of the two groups. The assignment was conducted by a medical secretary. To minimize bias based on patient expectations, allocation was not revealed to the patients (before the office hysteroscopy). They were informed about the scheduled time and place and received general information about the gynaecological procedure.

\section{Office hysteroscopy procedure}


The office hysteroscopy procedure and materials used during the procedure were identical in both groups, with the same experienced gynaecologist treating all patients. The standard appointment timeframe was 45 min. Patients were advised to take standard doses of Nonsteroidal anti-inflammatory drugs (NSAIDs)

(400 mg Ibuprofen) around 1 hour before their scheduled appointment.

Miniature hysteroscopies (CAMPO TROPHYSCOPE $®$ ) were used. All patients received local anaesthesia with a paracervical block of $6 \mathrm{ml}$ of mepivacaine $10 \mathrm{mg} / \mathrm{ml}$ in the cervix.

The choice of distension was normal saline [12]. With the patient in a dorsal lithotomy position, a vaginoscopy without the use of a vaginal speculum and cervical tenaculum was performed. The hysteroscopy was connected to a camera and a monitor. Depending on the findings, the following procedures were performed as required: biopsies, polypectomies, or myomectomies, using the same hysteroscope.

\section{Doula intervention group}

A doula accompanied the participants throughout the procedure, starting from the moment the patient registered at the front desk and ending when they left the clinic. In line with the elements of doula support in the childbirth context [13], the doula in the present study aimed to provide: a) attention to physical comfort, b) emotional support (praise, reassurance, encouragement, and continuous presence); c) information sharing (nonmedical advice, explanation of policies and procedures, anticipatory guidance) and d) advocacy (facilitation of communication between the woman and hospital staff to assist in making informed decisions). The doula was qualified, had 12 years of experience as a doula, and was employed as an assistant nurse at the clinic. To avoid spill-over effects, she was not attending to patients in the control group.

\section{Routine care control group}

The control group received basic support from an assistant nurse during the office hysteroscopy and were provided a comfortable and safe environment with acoustic, thermal, and visual comfort. The basic support from the nurse assistant had the main focus to facilitate the gynaecological procedure. This was different from the doula support, which focused on the patient's needs and participation.

\section{Sample size}

A total of 28 women were included, 12 in the Doula intervention group and 16 in the routine care group.

\section{Outcomes}

Background data were collected by the gynaecologist or a research assistant at the start of the appointment, including age, body mass index, the number of vaginal deliveries, educational achievement level, and history of endometrial surgery (curettage and/or hysteroscopy), diabetes, age, previous curettage, dyspareunia, severe dysmenorrhea, and previous hysteroscopy experience. Cervical stenosis 
was classified based on localization: stenosis of external cervical ostium (ECO; type I); stenosis of the distal third of cervical channel and the internal cervical ostium (ICO; type II); stenosis of the ICO (type III), and combined stenosis of ECO and ICO (type IV))[14]. Outcome data were collected at the start of the office hysteroscopy, and at different phases during the procedure. STAI-s post-procedure were collected in the same room immediately after the hysteroscopy.

\section{Feasibility}

Feasibility was evaluated by three outcomes:

1) Number of successful (access to and visualization of the entire uterine cavity was possible during the same procedure), partially successful (access to uterine cavity possible, but the entire uterine cavity could not be examined) or failed (access to uterine cavity was not possible) hysteroscopies.

2) Duration of the procedure.

3) Complications (bleedings, false tracks, uterine perforation or cervical tears, or reactions to mepivacain (numbness and tingling of the lips or tongue, nervousness, dizziness, blurred vision, tremors, drowsiness, convulsions, sweating, unconsciousness or respiratory arrest).

\section{Anxiety}

The Spielberg State-Trait Anxiety Inventory (STAI) was used to measure the patients' anxiety before and after the office hysteroscopy [2,15-16]. The STAI is a widely used measure, validated among perinatal women, where it has shown acceptable sensitivity, specificity, and predictive validity [17]. The assessment consists of two subscales, the STAI-S (state anxiety) and the STAI-T (anxiety as a general trait). Each subscale has 20 items with each item rated using a 4-point Likert scale and a score $>40$ as the suggested cut off for clinically significant anxiety [18]. For this study, to minimize the time and number of questions asked to the participants, only the STAI-S was used to measure the situational anxiety pre- and postprocedure.

\section{Pain}

The Numeric Rating Scale (NRS), an instrument for self-reported pain intensity, was used. The patient reports a number between 0 and 10, where 10 stands for worst possible pain, 0 indicates no pain. The NRS is similar to the commonly used Visual Analog Scale (VAS) but is less influenced by non-pain intensity factors [19]. Pain intensity was assessed via NRS 0-10 at four points in time during the procedure:

1. Introduction of hysteroscopy into the vagina.

2. Progression through the cervical canal up to the internal uterine orifice.

3. Inspection of the uterine cavity.

4. Performance of the endometrial biopsy/polypectomy etc. if required. 
Previous well-defined limits for unacceptable pain were used [20]. Pain was considered unacceptable if severe pain was reported during the procedure (NRS $\geq 7$ ). In these cases, the office hysteroscopy was discontinued. The pain variable was also dichotomized, defined as $>4=$ moderate-to-severe pain [21].

\section{Statistical analyses}

The sample size was planned in line with the rule of thumb for pilot studies [22], suggesting 12 subjects in each group.

Descriptive statistics were used to describe the clinical and background variables. For the continuous variables pain (NRS) and anxiety (STAI-S), differences between groups were analysed using analysis of variance (ANOVA). Data for the STAI-S was approximately normally distributed according to histograms and Shapiro Wilk's test of normality. The dichotomized pain variable (NRS) was analysed using the chisquare test. A p-value of $\leq 0.05$ was set as the limit for statistical significance in all tests. IBM SPSS Statistics version 25.0 was used for all statistical analyses.

\section{Ethical considerations}

To minimize the risk of harm, the office hysteroscopy was discontinued if pain increased above predefined limits. All participants were informed that participation was voluntary and declining to or withdrawing from the study would not affect their treatment at the clinic. The study was approved by the Regional Ethics Review Board in Gothenburg, registration number 840-18.

\section{Results}

Twenty-eight women participated in the study with a median age of 43.5 (range 21-73) years. The main reason for the exclusion of potential participants was that they could not understand written Swedish. The flow diagram of participants through the study is shown in Figure 1.

A total of 28 women were included. There were no significant differences between the two groups regarding baseline characteristics (Table 1) and indications for office hysteroscopy (Table 2).

Polyps were identified in 4 cases (33\%) in the doula group and $6(40 \%)$ in the control group, there was 1 $(8.3 \%)$ case of myoma and $2(16.7 \%)$ cases of uterine malformation in the doula group. Also, 3 (25\%) cases in the doula group and $5(33.3 \%)$ in the control group had retained Intrauterine Contraceptive Devices (IUCD) and there were $2(16.7 \%)$ normal hysteroscopies in the doula group and $4(26.7 \%)$ in the control group

\section{Feasibility outcomes}

In two cases ( $\mathrm{n}=2$ ) one in each group, the office hysteroscopy was classified as non-successful. In the doula group, one office hysteroscopy could not be performed due to pain at the entrance to the cervix, so a blind endometrial biopsy was performed. In the other case, the polyp recession could be started but had 
to be stopped due to pain and bleeding. The first case was scheduled for a hysteroscopy under general anaesthesia, whereas in the second case, a second office hysteroscopy was performed, and the polyp was completely resected.

In the doula group, three hysteroscopies were classified as partially successful, in these cases, biopsies were performed and the base of the polyp sectioned even though the polyp could not be fully removed. Ultrasound controls after three months were normal and did not require further interventions.

There were no significant differences between the groups regarding cervical stenosis. Cervical stenos type II was found in one woman in each group and one cervical stenos type III was found in the control group.

The mean duration of the procedure was 18.6 minutes (range 4-40) in the doula group and 18.9 minutes (range 8-40) in the control group (Table 2), with no significant difference between groups. There were no procedure-related complications and no participant reported side-effects of mepivacain.

\section{Effectiveness}

There were no differences in pain outcome between the groups, see Table 4. For the anxiety outcome STAI-S, both groups had high, comparable levels of state anxiety before the procedure. After the procedure, the doula group showed slightly increased state anxiety, while the control group showed slightly decreased anxiety. There was a significant difference between groups favouring the control group when comparing STAI-S mean score post-procedure, but not when analysing mean change across groups (Table 2 and Figure 1). There were no significant differences in post-procedural anxiety and age, duration of the procedure, the reason for office hysteroscopy, or parity.

\section{Discussion}

This pilot study suggests that feasibility of doula support was acceptable (success rates, complications, duration of procedure) but the results indicate poor effectiveness regarding pain and anxiety. Unexpectedly, all participants presented high levels of state anxiety both before and after the procedure but relatively low pain ratings.

There were two cases one in the doula group and one in the control group where the office hysteroscopy was unsuccessful, but the percentage of successful office hysteroscopies was above $90 \%$ in both groups, which is comparable with data from previous studies $[14,23]$.

The mean duration of the office hysteroscopy was similar in the two groups, around 18 minutes. [12].

Both groups showed low and comparable pain ratings overall, suggesting that the setting may have been beneficial to minimize pain perception. State anxiety pre to post-procedure increased slightly in the doula group and decreased very little in the control group (Table 2). This might reflect high levels of anxiety in the study population [24-25], which we did not measure in our attempt to minimize the questionnaires. 
High pre- and post-procedural anxiety highlight a vulnerability in the population that is relevant to address. For future investigations of human support in office hysteroscopy, we suggest that trait anxiety should be added as a variable. Qualitative studies might also reveal important aspects.

We did not systematically evaluate patient satisfaction, which is a limitation of this study. However, reports from staff and patient's spontaneous comments after the procedure indicated that some aspects of doula support are positive. The gynaecologist and other staff members expressed appreciation for the doula's presence in examination rooms and that doulas created a more patient-centred clinical experience. In line with our experiences, a study [9] described that a gynaecologist and staff reported that doulas allowed them greater freedom to focus on technical aspects of the procedure without sacrificing patient comfort.

Another study evaluating doula support in the surgical management of miscarriage showed that $72 \%$ of the study participants reported that it was important to have someone with them during the procedure, but the support person did not have to be a doula [26]. In line with these results, the lack of a doula effect in our study could be due to high-quality routine care, including adequate psychosocial support from the ordinary gynaecological team.

There is also a risk of bias with the STAI-S measurement due to the Covid-19 pandemic. During March and April 2020, patients reported that they were more nervous and anxious about coming to the hospital. They also reported difficulties in differentiating between their anxiety related to the procedure, and to their overall distress related to Covid-19, similar to that reported in other studies [28-29].

\section{Conclusion}

While there was no indication of the effectiveness of doulas on pain or anxiety during office hysteroscopy, pre- and post-procedural anxiety among the patients may be higher than previously believed. Moreover, high anxiety levels may be more of an issue than the pain during the procedure, therefore, it is recommended that trait and state anxiety in different hysteroscopy populations are investigated further.

\section{Abbreviations}

ECO: external cervical ostium

ICO: Internal cervical ostium

IUCD: Intrauterine Contraceptive Devices

NRS: Numeric Rate Scale

NSAIDs: Nonsteroidal anti-inflammatory drugs 
STAI: Spielberg State-Trait Anxiety Inventory-S

TENS: Transcutaneous Electric Nerve Stimulation

VAS: Visual Analog Scale

\section{Declarations}

\section{Ethics approval and consent to participate}

The study was approved by the Regional Ethics Review Board in Gothenburg, registration number 840-18.

\section{Availability of data and materials}

The dataset analysed during the current study is available from the corresponding author on reasonable request.

\section{Competing interests}

The authors report that there is no conflict of interest.

\section{Funding}

The authors received no specific funding for this work

\section{Contributions}

$\mathrm{RM}, \mathrm{LD}$ and $\mathrm{JH}$ designed the trial. RM ran the trial and recruited patients. RM, LD and JH analysed the data. RM,LD and JH and LS interpreted the data and wrote the paper.

\section{Corresponding author}

Correspondence to Rocio Montejo: rocio.montejo.rodriguez@vgregion.se

\section{Acknowledgments}

We thank the patients and the staff who participated in the study. We are most grateful to Etab El Metowri, Louise Karlsson and Sinaria Baban at the Gynecology Department and to Maria Nyström at the Pain Clinic, Angered Hospital.

\section{References}

1. Siristatidis C. and C. Chrelias."Feasibility of office hysteroscopy through the "see and treat technique" in private practice: a prospective observational study. Arch Gynecol Obstet. 2011;283(4):819-23. 
2. Gupta JK, Clark TJ, More S. \& H. Pattison. Patient anxiety and experiences associated with an outpatient "one-stop" "see and treat" hysteroscopy clinic. Surg Endosc. 2004;18:1099-104.

3. Lisón JF, Amer-Cuenca JJ, Piquer-Martí S, Benavent-Caballer V, Biviá-Roig G. Marín- Buck A. Transcutaneous Nerve Stimulation for Pain Relief During Office Hysteroscopy: A Randomized Controlled Trial. Obstet Gynecol. 2017;129(2):363-70.

4. Amer-Cuenca JJ, Marín-Buck A, Vitale SG, La Rosa VL, Caruso S, Cianci A. \& J.F. Lisón.Nonpharmacological pain control in outpatient hysteroscopies. Minim Invasive Ther Allied Technol. 2019; 1-10.

5. Angioli R, De Cicco Nardone C, Plotti F, et al. Use of music to reduce anxiety during office hysteroscopy: prospective randomized trial. J Minim Invasive Gynecol. 2014;21:454-9.

6. Mak N, Reinders IMA, Slockers SA, Westen EHMN, Maas JWM. \& M. Y. Bongers. The effect of music in gynaecological office procedures on pain, anxiety and satisfaction: a randomized controlled trial. Gynecol Surg. 2017;14:14.

7. Kayne MA, Greulich MB, Albers LL. Doulas: an alternative yet complementary addition to care during childbirth. Clin Obstet Gynecol. 2001;44:692-703.

8. Chor J, Hill B, Martins S, Mistretta S, Patel A. \& M. Gilliam.Doula support during first-trimester surgical abortion: a randomized controlled trial. Am J Obstet Gynecol. 2015;212:45.e1-6.

9. Chor J, Lyman P, Ruth J, Patel A. \& M. Integrating Doulas Into First-Trimester Abortion Care: Physician, Clinic Staff, and Doula Experiences. J Midwifery Womens Health. 2018;63:53-7.

10. Chor J, Lyman P, Tusken M, Patel A, Gilliam M. Women's experiences with doula support during firsttrimester surgical abortion: a qualitative study. Contraception. 2016;93:244-8.

11. Eldridge SM, Chan CL, Campbell MJ, Bond CM, Hopewell S, Thabane L, Lancaster GA. \& P. c. group CONSORT 2010 statement: extension to randomised pilot and feasibility trials. Pilot Feasibility Stud. 2016;2:64.

12. Campo R, Santangelo F, Gordts S, Di Cesare C, Van Kerrebroeck H, De Angelis MC. \& A. Di Spiezio Sardo. Outpatient hysteroscopy. Facts Views Vis Obgyn. 2018;10:115-22.

13. Amram NL, Klein MC, Mok H, Simkin P, Lindstrom K, Grant J. How birth doulas help clients adapt to changes in circumstances, clinical care, and client preferences during labor. J Perinat Educ. 2014;23(2):96-103. doi:10.1891/1058-1243.23.2.96.

14. Bettocchi S, Bramante S, Bifulco G, Spinelli M, Ceci O, Fascilla FD. \& A. Di Spiezio Sardo. Challenging the cervix: strategies to overcome the anatomic impediments to hysteroscopy: analysis of 31,052 office hysteroscopies. Fertil Steril. 2016;105:e16-7.

15. Kokanali MK, Cavkaytar S, Guzel A, Topçu HO, Eroğlu E, Aksakal O. \& M. Doğanay Impact of preprocedural anxiety levels on pain perception in patients undergoing office hysteroscopy. J Chin Med Assoc. 2014;77:477-81.

16. Spielberger CD, Gorsuch RL. Manual for the State-Trait Anxiety Inventory (Form Y). Palo Alto: Consulting Psychologists Press; 1983. 
17. Meades R. \& S. Ayers.Anxiety measures validated in perinatal populations: a systematic review. J Affect Disord. 2011;133:1-15.

18. Grant KA, McMahon C. \& M. P. Austin. Maternal anxiety during the transition to parenthood: a prospective study. J Affect Disord. 2008;108:101-11.

19. Thong ISK, Jensen MP, Miró J, Tan G. The Validity of Pain Intensity Measures: What Do the NRS, VAS, VRS, and FPS-R Measure? Scand J Pain. 2018;18(1):99-107.

20. de Freitas Fonseca M, Sessa FV, Resende JA, Guerra CG, Andrade CM. \& C. P. Crispi. Identifying predictors of unacceptable pain at office hysteroscopy. J Minim Invasive Gynecol. 2014;21:586-91.

21. Gerbershagen HJ, Rothaug J, Kalkman CJ, Meissner W. Determination of moderate-to-severe postoperative pain on the numeric rating scale: a cut-off point analysis applying four different methods. Br J Anaesth. 2011;107:619-26.

22. Julious SA. Sample size of 12 per group rule of thumb for a pilot study. Pharmaceut Stat. 2005;4:287-91.

23. Bradley LD, Widrich T. State-of-the-art flexible hysteroscopy for office gynecologic evaluation. J Am Assoc Gynecol Laparosc. 1995;2:263-7.

24. Cicinelli E. Hysteroscopy without anesthesia: review of recent literature. J Minim Invasive Gynecol. 2010;17(6):703-8.

25. Cicinelli E, Rossi AC, Marinaccio M, Matteo M, Saliani N. \& R. Tinelli. Predictive factors for pain experienced at office fluid minihysteroscopy. J Minim Invasive Gynecol. 2007;14:485-8.

26. Wilson SF, Gurney EP, Sammel MD \& C. A. Schreiber. Doulas for surgical management of miscarriage and abortion: a randomized controlled trial. Am J Obstet Gynecol. 2017;216, 44.e1-44.e6.

27. Gagliardi AR, Green C, Dunn S, Grace SL, Khanlou N. \& D. E. Stewart. How do and could clinical guidelines support patient-centred care for women: Content analysis of guidelines. PLoS One. 2019;14:e0224507.

28. Rajkumar RP. COVID-19 and mental health: A review of the existing literature. Asian J Psychiatr. 2020;52:102066.

29. Röhr S, Müller F, Jung F, Apfelbacher C, Seidler A. \& S. G. Riedel-Heller. Psychosocial Impact of Quarantine Measures During Serious Coronavirus Outbreaks: A Rapid Review. Psychiatr Prax. 2020;47:179-89.

\section{Tables}


Table 1

Baseline and clinical characteristics of participants

\begin{tabular}{|lllll|}
\hline & $\begin{array}{l}\text { Doula group } \\
\mathbf{n = 1 2}\end{array}$ & $\begin{array}{l}\text { Control group } \\
\mathbf{n = 1 6}\end{array}$ & $\begin{array}{l}\text { Total } \\
\mathbf{n = 2 8}\end{array}$ & P-value $^{\mathbf{1}}$ \\
\hline Age, mean & $47.5 \pm 13.6$ & $40.3 \pm 12.1$ & & 0.32 \\
\hline Parity, mean & $1.6 \pm 1.3$ & $1.18 \pm 1.1$ & 0.29 \\
\hline Suspected of polyp & $7(46.7 \%)$ & $8(53.3 \%)$ & $15(100 \%)$ & \\
\hline Abnormal uterine bleeding & $1(100 \%)$ & 0 & $1(100 \%)$ & \\
\hline Remove of IUCD & $2(20 \%)$ & $8(80 \%)$ & $10(100 \%)$ & \\
\hline $\begin{array}{l}1 \text { Values are from comparisons between groups, analyzed with ANOVA (continuous variables). IUCD }= \\
\text { Intrauterine Contraceptive Devices }\end{array}$ & & \\
\hline
\end{tabular}


Table 2

Principal outcome measures showing group comparisons

\begin{tabular}{|c|c|c|c|}
\hline & Doula group & Control group & $\begin{array}{l}\text { p- } \\
\text { value }^{1}\end{array}$ \\
\hline Successful office hysteroscopy & $11(92 \%)$ & $15(94 \%)$ & 0.84 \\
\hline Partially successful office hysteroscopy & $3(8 \%)$ & 0 & 0.256 \\
\hline Mean duration of procedure, minutes & 18.5 (SD 13.7) & $\begin{array}{l}18.8(\mathrm{SD} \\
11.8)\end{array}$ & 0.38 \\
\hline VAS vaginoscopy, mean & 0 & $0.2(\mathrm{SD} 0.6)$ & 0.21 \\
\hline VAS cervix, mean & $0.42(S D 0.7)$ & 1.3(SD 1.6) & 0.091 \\
\hline VAS uterine cavity, mean & 1.7(SD 1.7) & $1.5(\mathrm{SD} 1.9)$ & 0.85 \\
\hline VAS treatment, mean & 3.2(SD 1.4) & 3.7(SD 2.6) & 0.671 \\
\hline Dichotomized pain VAS $>4$, cervix, $n$ & $0 / 12(0 \%)$ & $1 / 16(6 \%)$ & 0.57 \\
\hline Dichotomized pain VAS $>4$, uterine cavity, $n$ & $1 / 11(9 \%)$ & $2 / 12(17 \%)$ & 0.67 \\
\hline Dichotomized pain VAS $>4$, treatment, $n$ & $1 / 5(20 \%)$ & $5 / 12(42 \%)$ & 0.56 \\
\hline STAI-S pre-procedure, mean score & $\begin{array}{l}45.41(\mathrm{SD} \\
10.22)\end{array}$ & 45.81(SD 6.2) & 0.90 \\
\hline STAI-S post-procedure, mean score & 48.58(SD 4.9) & 44.1(SD 5.4) & 0.033 \\
\hline $\begin{array}{l}\text { Mean change pre to post-procedure STAI-S, mean } \\
\text { score }\end{array}$ & 3.3(SD 11.6) & $-1.5($ SD 5.1) & 0.205 \\
\hline
\end{tabular}

\section{Figures}




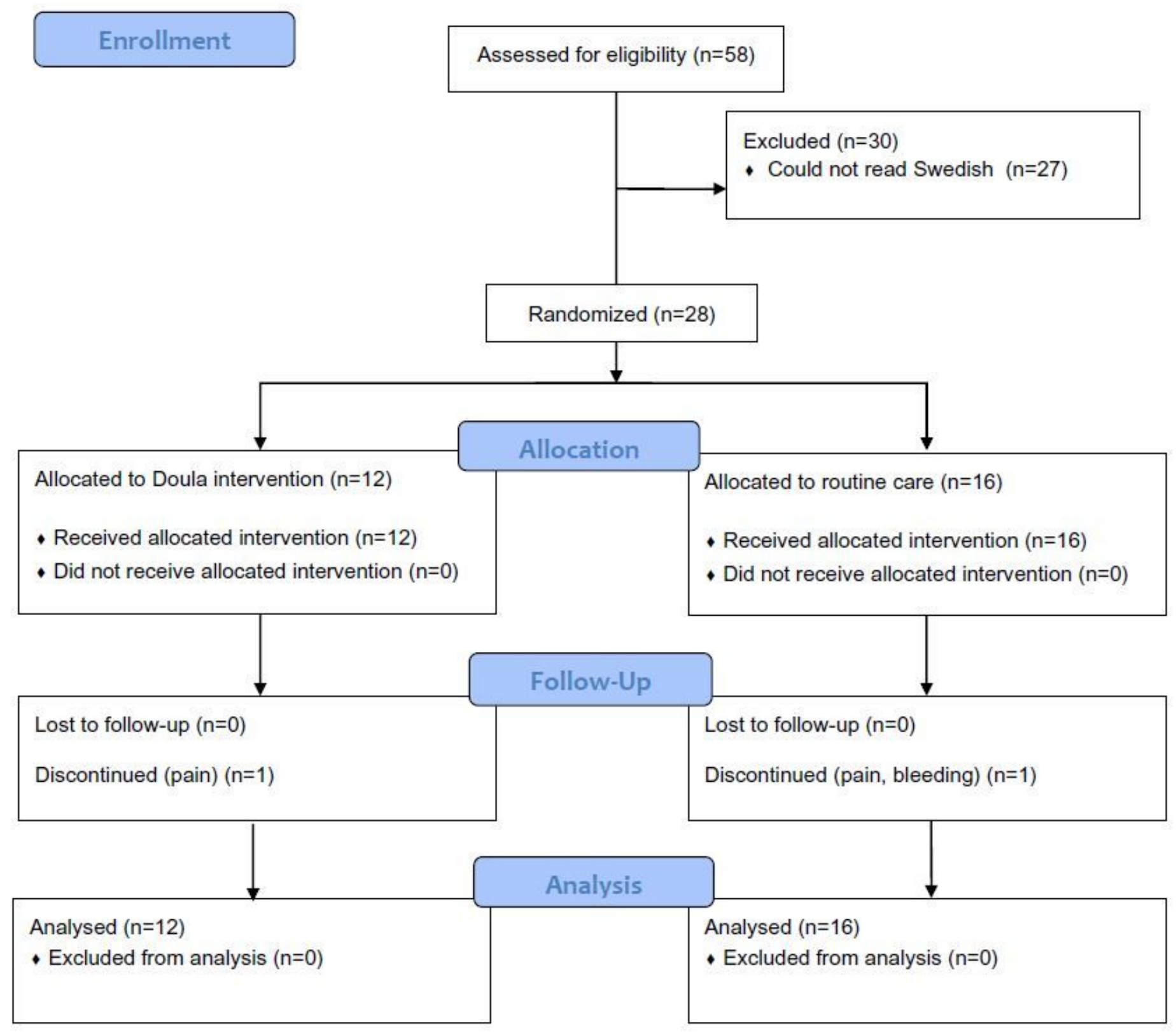

\section{Figure 1}

Twenty-eight women participated in the study with a median age of 43.5 (range 21-73) years. The main reason for the exclusion of potential participants was that they could not understand written Swedish. The flow diagram of participants through the study is shown in Figure 1. 


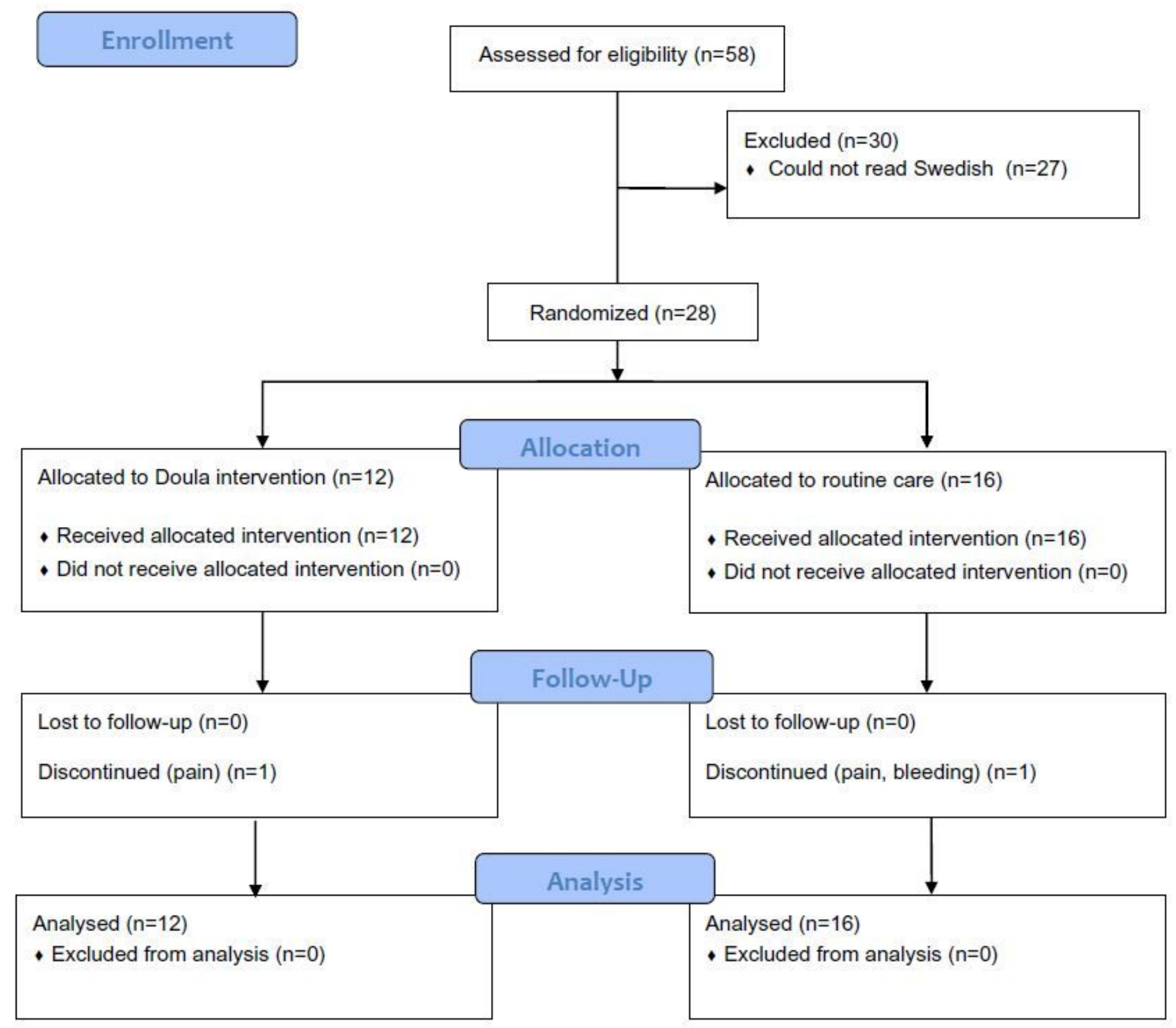

Figure 1

Twenty-eight women participated in the study with a median age of 43.5 (range 21-73) years. The main reason for the exclusion of potential participants was that they could not understand written Swedish. The flow diagram of participants through the study is shown in Figure 1.

\section{Supplementary Files}

This is a list of supplementary files associated with this preprint. Click to download.

- CONSORTextensionforPilotandFeasibilityTrialsChecklist.doc

- CONSORTextensionforPilotandFeasibilityTrialsChecklist.doc 